\title{
Dual weighted residual based error control for nonstationary convection-dominated equations: potential or ballast?
}

\author{
M. P. Bruchhäuser*, K. Schwegler, M. Bause ${ }^{\dagger}$ \\ Helmut Schmidt University, Faculty of Mechanical Engineering, \\ Holstenhofweg 85, 22043 Hamburg, Germany
}

\begin{abstract}
Even though substantial progress has been made in the numerical approximation of convection-dominated problems, its major challenges remain in the scope of current research [14. In particular, parameter robust a posteriori error estimates for quantities of physical interest and adaptive mesh refinement strategies with proved convergence are still missing. Here, we study numerically the potential of the Dual Weighted Residual (DWR) approach applied to stabilized finite element methods to further enhance the quality of approximations. The impact of a strict application of the DWR methodology is particularly focused rather than the reduction of computational costs for solving the dual problem by interpolation or localization.
\end{abstract}

Keywords: Convection-dominated problems, stabilized finite element methods, space-time adaptivity, goal-oriented a posteriori error control, Dual Weighted Residual method, duality techniques

\section{Introduction}

In their recent review paper 14 the authors nicely survey the current state of research in the numerical approximation of convection-dominated equations and incompressible flow. These problems with prominent applications in many branches of technology have strongly attracted researchers' interest not just since the pioneering works of the 1980's (cf., e.g., [10, 12]). The introduction of various families of linear and nonlinear residual-based stabilization techniques and of algebraic stabilization techniques, usually referred to as algebraic flux correction schemes, are regarded as milestones in the development of discretization techniques that are able to reduce spurious nonphysical oscillations close to sharp layers of convection-dominated problems; cf. 15] for a comparative study of those techniques. For a general review of those methods as well as a list of references we refer to, e.g., [14, 17]. In [14, the authors further identify

\footnotetext{
*bruchhaeuser@hsu-hh.de

†bause@hsu-hh.de
} 
numerous problems that are still unresolved in this field of research. In particular, the non-availabilty of parameter-robust a posteriori error estimates for quantities of physical interest and in general situations is stressed. Moreover, the authors point out that adaptive mesh refinement strategies that are based on such a posteriori error estimates and guarantee convergence in appropriate norms are desirable and indespensable for further improvement.

One possible technique for those adaptive strategies is the DWR method ([7, 8, 3]), where the error is estimated in an arbitrary user-chosen goal quantity of physical interest. The DWR approach relies on a space-time variational formulation of the discrete problem and uses duality techniques to find rigorous a posteriori error estimates, obtained through the approximation of an additional dual problem. Early studies for adaptive mesh refinement applied to various stationary stabilized equations date back to the end of the last century; cf. 8, Section 3.3 and 8] for a brief overview and further literature. For the nonstationary Navier-Stokes equations the DWR approach was applied together with local projection stabilization (LPS) in [9]. In [1] an adaptive algorithm in time is presented for convection-dominated problems, where the time step control uses a post-processed solution.

In this work, we study numerically the potential of combining the DWR approach with SUPG ([12, 10]) stabilized finite element methods for the efficient and reliable approximation of nonstationary convection-dominated problems. Here, a first dualize and then stabilize principle is applied; cf. Sect. 3. For the approximation of the dual problem higher-order schemes are used, due to the recently received results for stationary convection-dominated problems in [11, where a comparative study to an approximation by higher-order interpolation was done. The presented numerical results illustrate the performance properties and robustness of the proposed algorithm with respect to a vanishing perturbation or diffusion parameter. Thereby, the potential of a DWR approach is demonstrated and it is shown that the DWR based adaptivity is not ballast for the approximation of convection-dominated problems.

This work is organized as follows. In Sect. 2 we present our model problem as well as the stabilized space-time discretization. A first dualize and then stabilize DWR approach as well as a localized error representation is presented in Sect. 3 . Finally, in Sect. 4 the results of our numerical experiments are presented.

\section{Model problem and stabilized space-time dis- cretizations}

In this work we consider the following convection-diffusion-reaction equation

$$
\begin{aligned}
\partial_{t} u-\nabla \cdot(\varepsilon \nabla u)+\vec{b} \cdot \nabla u+\alpha u & =f \quad \text { in } \Omega \times I, \\
u & =g_{D} \quad \text { on } \partial \Omega \times I, \\
u(0) & =u_{0} \quad \text { in } \Omega,
\end{aligned}
$$

where $\Omega \subset \mathbf{R}^{d}$, with $d=2$ or $d=3$ is a polygonal or polyhedral bounded domain with Lipschitz boundary $\partial \Omega$ and $I=(0, T), T>0$, is a bounded domain in time. To ensure the well-posedness of problem 2.1 we assume that $0<\varepsilon \leq 1$ is a constant diffusion coefficient, $\vec{b} \in \vec{H}^{1}(\Omega) \cap \vec{L}^{\infty}(\Omega)$ is the flow field or convection tensor, $\alpha \in L^{\infty}(\Omega)$ is the reaction coefficient, $u_{0} \in H_{0}^{1}(\Omega)$ is a given initial 
condition, $f \in L^{2}\left(I ; L^{2}(\Omega)\right)$ is a given source of the unknown scalar quantity $u$ and $g \in L^{2}\left(I ; H^{\frac{1}{2}}(\partial \Omega)\right)$ is a given function specifying the Dirichlet boundary condition. Furthermore, we assume that the conditions $\nabla \cdot \vec{b}(\vec{x})=0$ and $\alpha(\vec{x}) \geq 0$ are fulfilled for all $\vec{x} \in \Omega$. Henceforth, for the sake of simplicity, we deal with homogeneous Dirichlet boundary conditions only. In our numerical examples in Sect. 4, we also consider more general boundary conditions; cf. also Remark 3.5 .

It is well known that problem (2.1) along with the above conditions admits a unique weak solution $u \in V:=\left\{v \in L^{2}\left(I ; H_{0}^{1}(\Omega)\right) \mid \partial_{t} v \in L^{2}\left(I ; H^{-1}(\Omega)\right)\right\}$ that satisfies the following variational formulation; cf., e.g. [17].

Find $u \in V$, satisfying $u(0)=u_{0}$, such that

$$
A(u)(\varphi)=F(\varphi) \quad \forall \varphi \in V,
$$

where the bilinear form $A: V \times V \rightarrow \mathbf{R}$ and the linear form $F: V \rightarrow \mathbf{R}$ are

$$
\begin{aligned}
A(u)(\varphi) & :=\sum_{n=1}^{N} \int_{I_{n}}\left\{\left(\partial_{t} u, \varphi\right)+a(u)(\varphi)\right\} \mathrm{d} t+\sum_{n=2}^{N}\left([u]_{n-1}, \varphi_{n-1}^{+}\right), \\
F(\varphi) & :=\int_{I}(f, \varphi) \mathrm{d} t .
\end{aligned}
$$

Here, $a(u)(\varphi):=(\varepsilon \nabla u, \nabla \varphi)+(\vec{b} \cdot \nabla u, \varphi)+(\alpha u, \varphi)$ and $(\cdot, \cdot)$ denotes the standard inner product of $L^{2}(\Omega)$. For the discretization in time we divide the time interval $I$ into not necessarily equidistant, left-open subintervals $I_{n}:=\left(t_{n-1}, t_{n}\right]$, with $n=1, \ldots, N$, where $0=t_{0}<t_{1}<\cdots<t_{N}=T$ with step size $\tau_{n}=t_{n}-t_{n-1}$ and $\tau=\max _{n} \tau_{n}$. Next, we introduce the time-discrete function spaces.

$$
\begin{aligned}
V_{\tau}^{c G(r)} & :=\left\{v \in C\left(\bar{I} ; H_{0}^{1}(\Omega)\right)|v|_{I_{n}} \in \mathbf{P}_{r}\left(\bar{I}_{n} ; H_{0}^{1}(\Omega)\right)\right\} \\
V_{\tau}^{d G(r)} & :=\left\{v \in L^{2}\left(I ; H_{0}^{1}(\Omega)\right)|v|_{I_{n}} \in \mathbf{P}_{r}\left(I_{n} ; H_{0}^{1}(\Omega)\right), v_{\tau}(0) \in L^{2}(\Omega)\right\}
\end{aligned}
$$

where $\mathbf{P}_{r}\left(\bar{I}_{n} ; H_{0}^{1}(\Omega)\right)$ denotes the space of all polynomials in time up to degree $r \geq 0$ on $I_{n}$ with values in $H_{0}^{1}(\Omega)$. For some function $v_{\tau} \in V_{\tau}^{d G(r)}$ we define the limits $v_{\tau}^{ \pm}$from above and below of $v_{\tau}$ as well as their jump at $t_{n}$ by

$$
v_{\tau, n}^{ \pm}:=\lim _{t \rightarrow t_{n} \pm 0} v_{\tau}(t), \quad\left[v_{\tau}\right]_{n}:=v_{\tau, n}^{+}-v_{\tau, n}^{-} .
$$

Using the discontinuous Galerkin method for the time discretization of the so called primal problem 2.2 leads to the following time-discrete variational approximation.

Find $u_{\tau} \in V_{\tau}^{d G(r)}$ such that

$$
A\left(u_{\tau}\right)\left(\varphi_{\tau}\right)+\left(u_{\tau, 0}^{+}, \varphi_{\tau, 0}^{+}\right)=F\left(\varphi_{\tau}\right)+\left(u_{0}, \varphi_{\tau, 0}^{+}\right) \quad \forall \varphi_{\tau} \in V_{\tau}^{d G(r)},
$$

with $A(\cdot)(\cdot)$ and $F(\cdot)$ being defined by (2.3) and $(2.4)$, respectively.

We note that the initial condition is incorporated into the variational problem. Next, we describe the Galerkin finite element approximation in space of the semidiscrete problem (2.7). We use Lagrange type finite element spaces of continuous functions that are piecewise polynomials. For the discretization 
in space, we consider a decomposition $\mathcal{T}_{h}$ of the domain $\Omega$ into disjoint elements $K$, such that $\bar{\Omega}=\cup_{K \in \mathcal{T}_{h}} \bar{K}$. Here, we choose the elements $K \in \mathcal{T}_{h}$ to be quadrilaterals for $d=2$ and hexahedrals for $d=3$. We denote by $h_{K}$ the diameter of the element $K$. The global space discretization parameter $h$ is given by $h:=\max _{K \in \mathcal{T}_{h}} h_{K}$. Our mesh adaptation process yields locally refined cells, which is enabled by using hanging nodes. We point out that the global conformity of the finite element approach is preserved since the unknowns at such hanging nodes are eliminated by interpolation between the neighboring 'regular' nodes; cf. [3]. On $\mathcal{T}_{h}$ we define the discrete finite element space by $V_{h}^{p, n}:=\left\{v \in V \cap C(\bar{\Omega})|v|_{K} \in Q_{h}^{p}(K), \forall K \in \mathcal{T}_{h},\right\}, n=1, \ldots, N$, where $Q_{h}^{p}(K)$ is the space of polynomials that are of degree less than or equal to $p$ with respect to each variable $x_{1}, \ldots, x_{d}$. By replacing $H_{0}^{1}(\Omega)$ in the definition of the semidiscrete function spaces $V_{\tau}^{c G(r)}$ and $V_{\tau}^{d G(r)}$ in $\sqrt{2.5}$ and $(2.6)$, respectively, by $V_{h}^{p, n}$, we obtain the fully discrete function spaces

$$
\begin{aligned}
V_{\tau h}^{c G(r), p}:=\left\{v_{\tau h} \in V_{\tau}^{c G(r)} \mid v_{\mid I_{n}} \in \mathbf{P}_{r}\left(\bar{I}_{n} ; V_{h}^{p, n}\right)\right\} \\
V_{\tau h}^{d G(r), p}:=\left\{v_{\tau h} \in V_{\tau}^{d G(r)} \mid v_{\mid I_{n}} \in \mathbf{P}_{r}\left(I_{n} ; V_{h}^{p, n}\right), v_{\tau h}(0) \in V_{h}^{p}\right\}
\end{aligned}
$$

with $V_{\tau h}^{c G(r), p} \subset V_{\tau}^{c G(r)}$ and $V_{\tau h}^{d G(r), p} \subset V_{\tau}^{d G(r)}$. We note that the spatial finite element space $V_{h}^{p, n}$ is allowed to be different on all intervals $I_{n}$ which is natural in the context of a discontinuous Galerkin approximation of the time variable and allows dynamic mesh changes in time. The fully discrete discontinuous in time scheme then reads as follows.

Find $u_{\tau h} \in V_{\tau h}^{d G(r), p}$ such that

$$
A\left(u_{\tau h}\right)\left(\varphi_{\tau h}\right)+\left(u_{\tau h, 0}^{+}, \varphi_{\tau h, 0}^{+}\right)=F\left(\varphi_{\tau h}\right)+\left(u_{0}, \varphi_{\tau h, 0}^{+}\right) \quad \forall \varphi_{\tau h} \in V_{\tau h}^{d G(r), p},
$$

with $A(\cdot)(\cdot)$ and $F(\cdot)$ being defined in $(2.3$ and $(2.4)$, respectively.

In this work we focus on convection-dominated problems with small difussion parameter $\varepsilon$. Then the finite element approximation needs to be stabilized in order to reduce spurious and non-physical oscillations of the discrete solution arising close to sharp fronts or layers. Here, we apply the streamline upwind Petrov-Galerkin method (for short SUPG); cf. [12, 10, 17, 15, 4, The stabilized variant of the fully discrete scheme 2.10 then reads as follows.

Find $u_{\tau h} \in V_{\tau h}^{d G(r), p}$ such that

$$
\begin{gathered}
A_{S}\left(u_{\tau h}\right)\left(\varphi_{\tau h}\right)+\left(u_{\tau h, 0}^{+}, \varphi_{\tau h, 0}^{+}\right)=F\left(\varphi_{\tau h}\right)+\left(u_{0}, \varphi_{\tau h, 0}^{+}\right) \quad \forall \varphi_{\tau h} \in V_{\tau h}^{d G(r), p}, \\
\text { with } A_{S}(u)(\varphi):=A(u)(\varphi)+S(u)(\varphi) \text { and stabilization terms }
\end{gathered}
$$

$$
\begin{aligned}
& S\left(u_{\tau h}\right)\left(\varphi_{\tau h}\right):=\sum_{n=1}^{N} \int_{I_{n}} \sum_{K \in \mathcal{T}_{h}} \delta_{K}\left(R\left(u_{\tau h}\right), \vec{b} \cdot \nabla \varphi_{\tau h}\right)_{K} \mathrm{~d} t \\
& +\sum_{n=2}^{N} \sum_{K \in \mathcal{T}_{h}} \delta_{K}\left(\left[u_{\tau h}\right]_{n-1}, \vec{b} \cdot \nabla \varphi_{\tau h, n-1}^{+}\right)_{K}+\sum_{K \in \mathcal{T}_{h}} \delta_{K}\left(u_{\tau h, 0}^{+}-u_{0}, \vec{b} \cdot \nabla \varphi_{\tau h, 0}^{+}\right)_{K}, \\
& R\left(u_{\tau h}\right):=\partial_{t} u_{\tau h}+\vec{b} \cdot \nabla u_{\tau h}-\nabla\left(\varepsilon \nabla u_{\tau h}\right)+\alpha u_{\tau h}-f .
\end{aligned}
$$

Remark 2.1. The proper choice of the stabilization parameter $\delta_{K}$ is an important issue in the application of the SUPG approach; cf., e.g., [13] and the discussion therein. For the situation of steady-state convection and reaction, an optimal error estimate for $\delta_{K}=\mathrm{O}(h)$ was derived in [13]. 


\section{A DWR approach and a posteriori error esti- mation}

The DWR method aims at the control of an error in an arbitrary user-chosen target functional $J$ of physical interest. To get an error representation with respect to this target functional, an additional dual problem has to be solved. Before we focus on the error representation, we introduce the dual problem of (2.2) whose derivation is based on the Euler-Lagrangian method of constrained optimization. For a detailed derivation we refer to [3, Chapter 6,9]. We note that here a so called first dualize and then stabilize principle is used, where the stabilization is applied to the discrete dual problem after its derivation via the Euler-Lagrangian method of constrained optimization; cf. [11, Remark 2].

For some given functional $J: V \rightarrow \mathbf{R}$ we consider solving

$$
J(u)=\min \{J(v), v \in V, \text { where } A(v)(\varphi)=F(\varphi) \forall \varphi \in V\} .
$$

We assume that the functional $J$ is Fréchet differentiable. i.e. $J^{\prime}(y) \in V^{\prime}$ for $y \in V$. For the derivation of the error representation we define the corresponding Lagrangian functional $\mathcal{L}: V \times V \rightarrow \mathbf{R}$ by

$$
\mathcal{L}(u, z):=J(u)+F(z)-A(u)(z)-\left(u(0)-u_{0}, z(0)\right),
$$

where we refer to $z \in V$ as the dual variable (or Lagrangian multiplier); cf. 3 . We determine a stationary point $\{u, z\} \in V \times V$ of $\mathcal{L}(\cdot, \cdot)$ by the condition $\mathcal{L}^{\prime}(u, z)(\psi, \varphi)$

$=0$, or equivalently by the system of equations

$$
\begin{aligned}
A^{\prime}(u)(\psi, z) & =J^{\prime}(u)(\psi) & \forall \psi \in V, \\
A(u)(\varphi) & =F(\varphi) & \forall \varphi \in V .
\end{aligned}
$$

The second of these equations, the $z$-component of the stationary condition, is just the given primal problem 2.2 , whereas the $u$-component of the stationary condition, is called the dual or adjoint equation with $A^{\prime}(u)(\psi, z)=$ $\int_{I}\left\{\left(\partial_{t} \psi, z\right)+a(\psi, z)\right\} \mathrm{d} t$ and $J^{\prime}(u)(\psi)=\int_{I}\{(j(u), \psi)\} \mathrm{d} t$ for some function $j(\cdot) \in L^{2}\left(I ; L^{2}(\Omega)\right)$. Applying integration by parts in time to the first term of $A^{\prime}$ and taking the condition $\nabla \cdot \vec{b}(\vec{x})=0$ into account (cf. Sect. 2 yields the representation $A^{*}(z)(\psi):=A^{\prime}(u)(\psi, z)=\int_{I}\left\{-\left(\partial_{t} z, \psi\right)+(\varepsilon \nabla z, \nabla \psi)-(\vec{b}\right.$. $\nabla z, \psi)+(\alpha z, \psi)\} \mathrm{d} t$. Finally, we find by using the proposed stabilized Galerkin discretization scheme 2.11 the following stabilized discrete dual problem.

$$
\begin{aligned}
& \text { Find } z_{\tau h} \in V_{\tau h}^{d G(r), p} \text { such that } \\
& A_{S}^{*}\left(z_{\tau h}\right)\left(\psi_{\tau h}\right)+\left(z_{\tau h, T}^{-}, \psi_{\tau h, T}^{-}\right)=J^{\prime}\left(u_{\tau h}\right)\left(\psi_{\tau h}\right) \quad \forall \psi_{\tau h} \in V_{\tau h}^{d G(r), p} .
\end{aligned}
$$


In (3.2), we put $A_{S}^{*}\left(z_{\tau h}\right)\left(\psi_{\tau h}\right):=A^{*}\left(z_{\tau h}\right)\left(\psi_{\tau h}\right)+S^{*}\left(z_{\tau h}\right)\left(\psi_{\tau h}\right)$ with

$$
\begin{aligned}
& A^{*}\left(z_{\tau h}\right)\left(\psi_{\tau h}\right):= \sum_{n=1}^{N} \int_{I_{n}}\left\{-\left(\partial_{t} z_{\tau h}, \psi_{\tau h}\right)-\left(\vec{b} \cdot \nabla z_{\tau h}, \psi_{\tau h}\right)+\left(\varepsilon \nabla z_{\tau h}, \nabla \psi_{\tau h}\right)\right\} \mathrm{d} t \\
&+\left(\alpha z_{\tau h}, \psi_{\tau h}\right)-\sum_{n=2}^{N}\left(\left[z_{\tau h}\right]_{n-1}, \psi_{\tau h, n-1}^{-}\right) \\
& S^{*}\left(z_{\tau h}\right)\left(\psi_{\tau h}\right):= \sum_{n=1}^{N} \int_{I_{n}} \sum_{K \in \mathcal{T}_{h}} \delta_{K}^{*}\left(R^{*}\left(z_{\tau h}\right),-\vec{b} \cdot \nabla \psi_{\tau h}\right)_{K} \mathrm{~d} t \\
&-\sum_{n=2}^{N} \sum_{K \in \mathcal{T}_{h}} \delta_{K}^{*}\left(\left[z_{\tau h}\right]_{n-1},-\vec{b} \cdot \nabla \psi_{\tau h, n-1}^{-}\right)_{K}+\sum_{K \in \mathcal{T}_{h}} \delta_{K}^{*}\left(z_{\tau h, N}^{-},-\vec{b} \cdot \nabla \psi_{\tau h, N}^{-}\right)_{K} \\
& R^{*}\left(z_{\tau h}\right):=-\partial_{t} z_{\tau h}-\vec{b} \cdot \nabla z_{\tau h}-\nabla\left(\varepsilon \nabla z_{\tau h}\right)+\alpha z_{\tau h}-j\left(u_{\tau h}\right) .
\end{aligned}
$$

To derive a representation of the error $J(e)=J(u)-J\left(u_{\tau h}\right)$ we need some abstract results. In order to keep this work self-contained we pare down to the key arguments of the DWR approach applied to the stabilized model problem. We follow the lines of [3. Chapter 6 and 9] and [6], where all of the proofs can be found. To start with, we need to extend the definition of the Lagrangian functional to arguments of $\left(V+V_{\tau h}^{d G(r), p}\right) \times V$. In the following we let $\mathcal{L}$ : $\left(V+V_{\tau h}^{d G(r), p}\right) \times V$ be defined by

$$
\mathcal{L}(u, z):=J(u)+F(z)-A(u)(z)-\sum_{n=2}^{N}\left([u]_{n-1}, z_{n-1}^{+}\right)-\left(u(0)-u_{0}, z(0)\right)
$$

Then it follows that

$$
\mathcal{L}_{u}(u, z)(\psi)+\mathcal{L}_{z}(u, z)(\varphi)=0 \quad \forall\{\psi, \varphi\} \in V \times V
$$

The discrete solution $\left\{u_{\tau h}, z_{\tau h}\right\} \in V_{\tau h}^{d G(r), p} \times V_{\tau h}^{d G(r), p}$ then satisfies

$$
\mathcal{L}_{u}\left(u_{\tau h}, z_{\tau h}\right)\left(\psi_{\tau h}\right)+\mathcal{L}_{z}\left(u_{\tau h}, z_{\tau h}\right)\left(\varphi_{\tau h}\right)=S\left(u_{k h}\right)\left(\varphi_{k h}\right)+S^{*}\left(z_{k h}\right)\left(\psi_{k h}\right)
$$

for all $\left\{\psi_{\tau h}, \varphi_{\tau h}\right\} \in V_{\tau h}^{d G(r), p} \times V_{\tau h}^{d G(r), p}$. For the defect of the discrete solution in the stationary condition 3.5 we use the notation

$$
\tilde{S}\left(x_{\tau h}\right)\left(y_{\tau h}\right):=S\left(u_{\tau h}\right)\left(\varphi_{\tau h}\right)+S^{*}\left(z_{\tau h}\right)\left(\psi_{\tau h}\right),
$$

with $x_{\tau h}:=\left\{u_{\tau h}, z_{\tau h}\right\} \in V_{\tau h}^{d G(r), p} \times V_{\tau h}^{d G(r), p}$ and $y_{\tau h}:=\left\{\psi_{\tau h}, \varphi_{\tau h}\right\} \in V_{\tau h}^{d G(r), p} \times$ $V_{\tau h}^{d G(r), p}$. To derive a representation of the error $J(u)-J\left(u_{\tau h}\right)$ we need the following abstract theorem that develops the error in terms of the Lagrangian functional.

Theorem 3.1. Let $X$ be a function space and $\mathcal{L}: X \rightarrow \mathbf{R}$ be a three times differentiable functional on $X$. Suppose that $x_{c} \in X_{c}$ with some ("continuous") function space $X_{c} \subset X$ is a stationary point of $\mathcal{L}$. Suppose that $x_{d} \in X_{d}$ with some ("discrete") function space $X_{d} \subset X$, with not necessarily $X_{d} \subset X_{c}$, is a Galerkin approximation to $x_{c}$ being defined by the equation

$$
\mathcal{L}^{\prime}\left(x_{d}\right)\left(y_{d}\right)=\tilde{S}\left(x_{d}\right)\left(y_{d}\right) \quad \forall y_{d} \in X_{d} .
$$


In addition, suppose that the auxiliary condition $\mathcal{L}^{\prime}\left(x_{c}\right)\left(x_{d}\right)=0$ is satisfied. Then there holds the error representation

$$
\mathcal{L}\left(x_{c}\right)-\mathcal{L}\left(x_{d}\right)=\frac{1}{2} \mathcal{L}^{\prime}\left(x_{d}\right)\left(x_{c}-y_{d}\right)+\frac{1}{2} \tilde{S}\left(x_{d}\right)\left(y_{d}-x_{d}\right)+\mathcal{R} \quad \forall y_{d} \in X_{d}
$$

where the remainder $\mathcal{R}$ is defined by $\mathcal{R}=\frac{1}{2} \int_{0}^{1} \mathcal{L}^{\prime \prime \prime}\left(x_{d}+s e\right)(e, e, e) \cdot s \cdot(s-1) \mathrm{d} s$, with the notation $e:=x_{c}-x_{d}$.

For the subsequent theorem we introduce the primal and dual residuals by

$$
\begin{array}{rlll}
\rho\left(u_{\tau h}\right)(\varphi) & :=F(\varphi)-A\left(u_{\tau h}\right)(\varphi)-\left(u_{\tau h, 0}^{+}-u_{0}, \varphi(0)\right) & \forall \varphi \in V \\
\rho^{*}\left(z_{\tau h}\right)(\psi) & :=J^{\prime}\left(u_{\tau h}\right)(\psi)-A^{*}\left(z_{\tau h}\right)(\psi)-\left(z_{\tau h, N}^{-}, \psi(T)\right) & \forall \psi \in V .
\end{array}
$$

Theorem 3.2. Suppose that $\{u, z\} \in V \times V$ is a stationary point of the Lagrangian functional $\mathcal{L}$ defined in (3.3) such that (3.4) is satisfied. Let $\left\{u_{\tau h}, z_{\tau h}\right\} \in$ $V_{\tau h}^{d G(r), p} \times V_{\tau h}^{d G(r), p}$ denote its Galerkin approximation being defined by (2.11) and (3.2), respectively, such that (3.5) is satisfied. Then there holds the error representation that

$$
J(u)-J\left(u_{\tau h}\right)=\frac{1}{2} \rho\left(u_{\tau h}\right)\left(z-\varphi_{\tau h}\right)+\frac{1}{2} \rho^{*}\left(z_{\tau h}\right)\left(u-\psi_{h}\right)+\mathcal{R}_{\tilde{S}}+\mathcal{R}_{J}
$$

for arbitrary functions $\left\{\varphi_{\tau h}, \psi_{\tau h}\right\} \in V_{\tau h}^{d G(r), p} \times V_{\tau h}^{d G(r), p}$, where the remainder terms are $\mathcal{R}_{\tilde{S}}:=\frac{1}{2} S\left(u_{\tau h}\right)\left(\varphi_{\tau h}+z_{\tau h}\right)+\frac{1}{2} S^{*}\left(z_{\tau h}\right)\left(\psi_{\tau h}-u_{\tau h}\right)$ and $\mathcal{R}_{J}:=$ $\frac{1}{2} \int_{0}^{1} J^{\prime \prime \prime}\left(u_{\tau h}+s \cdot e\right)(e, e, e) \cdot s \cdot(s-1) \mathrm{d} s$, with $e=u-u_{\tau h}$.

In the error respresentation (3.8) the continuous solution $u$ is required for the evaluation of the dual residual. The following theorem shows the equivalence of the primal and dual residual up to a quadratic remainder. This observation will be used below to find our final error respresentation in terms of the goal quantity $J$ and a suitable linearization for its computational evaluation or approximation, respectively.

Theorem 3.3. Under the assumptions of Thm. 3.2, and with the definitions (3.6) and (3.7) of the primal and dual residual, respectively, there holds that

$\rho^{*}\left(z_{\tau h}\right)\left(u-\psi_{\tau h}\right)=\rho\left(u_{\tau h}\right)\left(z-\varphi_{\tau h}\right)+S\left(u_{\tau h}\right)\left(\varphi_{\tau h}-z_{\tau h}\right)+S^{*}\left(z_{\tau h}\right)\left(u_{\tau h}-\psi_{\tau h}\right)+\Delta \rho_{J}$,

for all $\left\{\psi_{\tau h}, \varphi_{\tau h}\right\} \in V_{\tau h}^{d G(r), p} \times V_{\tau h}^{d G(r), p}$, where the remainder term is given by $\Delta \rho_{J}:=-\int_{0}^{1} J^{\prime \prime}\left(u_{\tau h}+s \cdot e\right)(e, e) \mathrm{d} s$ with $e:=u-u_{\tau h}$.

In a final step we combine the results of the previous two theorems to get a localized approximation of the error that is then used for the design of the adaptive algorithm. We note that the final result 3.9 is a slight modification of Thm. 5.2 for the nonstationary Navier-Stokes equations stabilized by LPS in [9]. The difference comes through using a first dualize and then stabilize approach as well as SUPG stabilization here. 
Theorem 3.4 (Localized error representation). Let the assumptions of Thm. 3.2 be satisfied. Neglecting the higher-order error terms, then there holds as a linear approximation the cell-wise error representation that

$$
\begin{aligned}
J(u)-J\left(u_{\tau h}\right) \doteq & \sum_{n=1}^{N} \int_{I_{n}} \sum_{K \in \mathcal{T}_{h}}\left\{\left(R\left(u_{\tau h}\right), z-\varphi_{\tau h}\right)_{K}-\delta_{K}\left(R\left(u_{\tau h}\right), \mathbf{b} \cdot \nabla \varphi_{\tau h}\right)_{K}\right. \\
& \left.-\left(E\left(u_{\tau h}\right), z-\varphi_{\tau h}\right)_{\partial K}\right\} \mathrm{d} t \\
& -\sum_{K \in \mathcal{T}_{h}}\left(u_{\tau h, 0}^{+}-u_{0}, z\left(t_{0}\right)-\varphi_{\tau h, 0}^{+}\right)_{K} \\
& -\sum_{n=2}^{N} \sum_{K \in \mathcal{T}_{h}}\left(\left[u_{\tau h}\right]_{n-1}, z\left(t_{n-1}\right)-\varphi_{\tau h, n-1}^{+}\right)_{K} \\
& +\sum_{K \in \mathcal{T}_{h}} \delta_{K}\left(u_{\tau h, 0}^{+}-u_{0}, \vec{b} \cdot \nabla \varphi_{\tau h, 0}^{+}\right)_{K} \\
& +\sum_{n=2}^{N} \sum_{K \in \mathcal{T}_{h}} \delta_{K}\left(\left[u_{\tau h}\right]_{n-1}, \vec{b} \cdot \nabla \varphi_{\tau h, n-1}^{+}\right)_{K} .
\end{aligned}
$$

The cell- and edge-wise residuals are defined by

$$
\begin{aligned}
R\left(u_{\tau h}\right)_{\mid K} & :=f-\partial_{t} u_{\tau h}+\nabla \cdot\left(\varepsilon \nabla u_{\tau h}\right)-\mathbf{b} \cdot \nabla u_{\tau h}-\alpha u_{\tau h}, \\
E\left(u_{\tau h}\right)_{\mid \Gamma} & :=\left\{\begin{array}{cl}
\frac{1}{2} \mathbf{n} \cdot\left[\varepsilon \nabla u_{\tau h}\right] & \text { if } \Gamma \subset \partial K \backslash \partial \Omega, \\
0 & \text { if } \Gamma \subset \partial \Omega,
\end{array}\right.
\end{aligned}
$$

where $\left[\nabla u_{\tau h}\right]:=\nabla u_{\tau h \mid \Gamma \cap K}-\nabla u_{v h \mid \Gamma \cap K^{\prime}}$ defines the jump of $\nabla u_{\tau h}$ over the inner edges $\Gamma$ with normal unit vector $\mathbf{n}$ pointing from $K$ to $K^{\prime}$.

Remark 3.5. (Nonhomogeneous Dirichlet boundary conditions) In the case of nonhomogeneous Dirichlet boundary conditions the following additional term has to be added to the error representation (3.9)

$$
\sum_{n=1}^{N} \int_{I_{n}}-\left(\left(g_{D}-\tilde{g}_{D, \tau h}\right), \varepsilon \nabla z \cdot \mathbf{n}\right)_{\partial \Omega} \mathrm{d} t,
$$

where the discrete function $\tilde{g}_{D, \tau h}$ is an appropriate finite element approximation of the extension $\tilde{g}_{D}$ in the sense that the trace of $\tilde{g}_{D}$ equals $g_{D}$ on $\partial \Omega$; cf. [11, 3].

\section{$4 \quad$ Numerical studies}

In this section we illustrate and investigate the performance properties of the proposed approach of combining the DWR method with SUPG stabilized finite element approximations of nonstationary convection-dominated problems. Therefore some general indications are needed. The error representation (3.9), written as

$$
J(u)-J\left(u_{\tau h}\right) \doteq \eta:=\sum_{n=1}^{N} \sum_{K \in \mathcal{T}_{h}} \eta_{K}^{n},
$$

depends on the discrete primal solution $u_{\tau h}$ as well as on the exact dual solution $z$. For solving the primal problem (2.1) we use the discontinuous in time scheme 
2.11) to get a discrete solution $u_{\tau h} \in V_{\tau h}^{d G(r), p}$. For the application of 4.1 in computations, the unknown dual solution $z$ has to be approximated, which results in an approximate error indicator $\tilde{\eta}$. This approximation cannot be done in the same finite element space as used for the primal problem, since this would result in an useless vanishing error representation $\tilde{\eta}=0$, due to Galerkin orthogonality. In contrast to the approximation by higher-order interpolation which is widespread used in the literature, cf. [3, 6, 9, we use an approximation by higher-order finite elements here. This is done due to the results in [11, where a comparative study between these two approaches is presented for steady convection-dominated problems. In this study the superiority of using higherorder finite elements was shown for an increasing convection dominance. Thus, we use for the discretization of the dual problem a finite element space that consists of polynomials in space and time that are at least of one polynomial degree higher than its primal counterpart, more precisely we compute a discrete dual solution $z_{\tau h} \in V_{\tau h}^{c G(r+1), p+1}$. For the now following example we briefly present our algorithm. For further details we refer to [16].

\section{Adaptive solution algorithm (Refining in space and time)}

Initialization: Set $i=0$ and generate the initial space-time slab $Q=\Omega \times I$ with $Q:=\cup_{n=1}^{N} Q_{n}=\cup_{n=1}^{N}\left(\Omega \times I_{n}\right)$.

1. Compute the primal and dual solution $u_{\tau h} \in V_{\tau h}^{d G(r), p}$ and $z_{\tau h} \in V_{\tau h}^{c G(r+1), p+1}$

2. Evaluate the a posteriori space-time error indicator $\tilde{\eta}:=\sum_{n=1}^{N} \sum_{K \in \mathcal{T}_{h}} \tilde{\eta}_{K}^{n}$. Mark the time intervals $I_{\tilde{n}}$ where $I_{\tilde{n}}$ belongs to the set of the time intervals $I_{n}$ according to $\theta_{\tau}$ percent of the worst indicators $\tilde{\eta}^{n}:=\sum_{K \in \mathcal{T}_{h}}\left|\tilde{\eta}_{K}^{n}\right|$. Mark those cells $\tilde{K}$ (of the respective spatial mesh of a space-time slab $Q_{n}$ ) for refinement that make up a certain fraction $\theta_{h}$ of the total error.

3. Check the stopping condition: If $\tilde{\eta}<$ tol is satisfied, then the adaptive solution algorithm is terminated.

4. Else, adapt the space-time slab $Q$, increase $i$ to $i+1$ and return to Step 1 .

For the implementation of the adaptive algorithm we use our DTM++ frontend software [16] that is based on the open source finite element library deal.II; cf. 2]. For measuring the accuracy of the error estimator, we will study the effectivity index

$$
\mathcal{I}_{\text {eff }}=\left|\frac{\tilde{\eta}}{J(u)-J\left(u_{h}\right)}\right|
$$

as the ratio of the estimated error $\tilde{\eta}$ of 4.1 over the exact error. Desirably, the index $\mathcal{I}_{\text {eff }}$ should be close to one.

\subsection{Example (Rotating hill with changing orientation).}

In this example we analyze the performance properties and the robustness of our algorithm with respect to the small perturbation parameter $\varepsilon$. We study problem 2.1 with the prescribed solution

$$
u(t, x, y):=\frac{\arctan (5 \pi(2 t-1))}{1+a_{0}\left(x-\frac{1}{2}-\frac{1}{4} \cos (2 \pi t)\right)^{2}+a_{0}\left(y-\frac{1}{2}-\frac{1}{4} \sin (2 \pi t)\right)^{2}},
$$


where $\Omega \times I:=(0,1)^{2} \times(0,1]$ and $a_{0}=50$. We choose the flow field $\mathbf{b}=(2,3)^{\top}$ and the reaction coefficient $\alpha=1.0$. The solution $\sqrt{4.3}$ is characterized by a counterclockwise rotating hill and designed in a way that the orientation of the hill changes its sign from negative to positive at the midpoint of the time interval $I$ at $t=0.5$. For the solution 4.3 the right-hand side function $f$ is calculated from the partial differential equation. Boundary conditions are given by the exact solution. Our target quantity is chosen to control the global $L^{2}$-error in space and time, given by

$$
J(u)=\frac{1}{\|e\|_{(0, T) \times \Omega}} \int_{I}(u, e) \mathrm{d} t, \quad \text { with }\|\cdot\|_{(0, T) \times \Omega}=\left(\int_{I}(\cdot, \cdot) \mathrm{d} t\right)^{\frac{1}{2}} .
$$

In our first test we investigate problem 2.1 for $\varepsilon=1$ and without any stabilization to verify our algorithm for a simple test case. In Fig. 4.1p we monitor the effectivity indices for uniform refinement. Here and in the following, $N_{\text {DoF }}^{\text {tot }}$ denotes the total number of degrees of freedom in space and time for one DWR loop while $N_{\text {DoF }}^{\max }$ denotes the maximum number of degrees of freedom of a spatial mesh used within one DWR loop. Furthermore, $N$ denotes the total number of space-time slabs $Q_{n}$ used for one DWR loop; cf. the adaptive solution algorithm at the beginning of this chapter. As we are using continuous finite elements of lowest order in space and discontinuous finite elements of lowest order in time (for short cG(1)-dG(0)), we refine globally once in space and twice in time after each step. For this uniform refinement the algorithm provides convincing and reliable values for the related effectivity indices; cf. the table in Fig. 4.1.

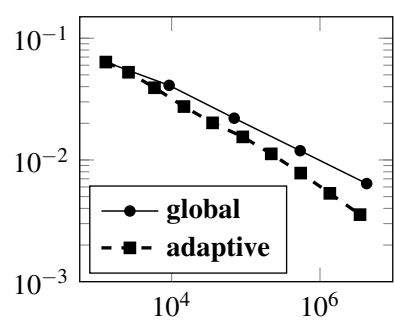

(a) Global $L^{2}$-error in space and time over $N_{\mathrm{DoF}}^{\text {tot }}$ for uniform and DWR adaptive mesh refinement.

\begin{tabular}{rrr}
\hline \hline \multicolumn{3}{c}{$\varepsilon=1$} \\
\hline$N_{\text {DoF }}^{\max }$ & $N$ & $\mathscr{I}_{\text {eff }}$ \\
\hline \hline 81 & 200 & 0.97 \\
289 & 800 & 0.98 \\
1089 & 3200 & 0.97 \\
4225 & 12800 & 0.97 \\
\hline
\end{tabular}

(b) Effectivity indices $\mathscr{I}_{\text {eff }}$ for uniform refinement.

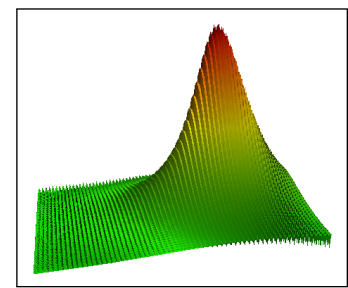

(c) Perturbed solution profile at $t_{n}=T$ on a uniform refined mesh with $N_{\text {DoF }}^{\text {tot }}=3328200$.

Figure 4.1: Comparison of global space-time $L^{2}$-errors, table for uniform refinement as well as perturbed solution profile.

In the sequel, all investigations are performed using SUPG stabilization in the sense of (2.11) for varying diffusion coefficients. Initially, in Fig. 4.1 a we compare the convergence behavior of the proposed DWR approach with a uniform mesh refinement strategy for $\varepsilon=10^{-6}$. The DWR based adaptive mesh adaptation is clearly superior to the uniform refinement in terms of accuray over the total number of degrees of freedom in space and time. In Table 4.1 we present selected effectivity indices of the proposed DWR approach applied to the stabilized approximation scheme 2.11) for different diffusion coefficients. For $\varepsilon=10^{-3}$ the effectivity indices nicely converge to one for an increasing number of the total degrees of freedom in space and time. For the more challenging case of $\varepsilon=10^{-6}$ the values of $\mathcal{I}_{\text {eff }}$ are still close to one, which confirms 


\begin{tabular}{rrrccc|rrrrrr}
\hline \hline \multicolumn{1}{c|}{$\varepsilon=10^{-3}$} & \multicolumn{6}{c}{$\varepsilon=10^{-6}$} \\
\hline$N_{\text {DoF }}^{\text {tot }}$ & $N_{\text {DoF }}^{\max }$ & \multicolumn{1}{c|}{$N$} & $J(e)$ & $|\tilde{\eta}|$ & $\mathcal{I}_{\text {eff }}$ & $N_{\text {DoF }}^{\text {tot }}$ & $N_{\text {DoF }}^{\max }$ & $N$ & $J(e)$ & $|\tilde{\eta}|$ & $\mathcal{I}_{\text {eff }}$ \\
\hline 17650 & 628 & 36 & $3.244 \mathrm{e}-02$ & $2.301 \mathrm{e}-02$ & 0.71 & 10974 & 617 & 22 & $4.761 \mathrm{e}-02$ & $2.335 \mathrm{e}-02$ & 0.491 \\
37728 & 961 & 54 & $2.381 \mathrm{e}-02$ & $9.258 \mathrm{e}-03$ & 0.39 & 22868 & 951 & 33 & $3.317 \mathrm{e}-02$ & $1.824 \mathrm{e}-02$ & 0.550 \\
86919 & 1464 & 81 & $1.839 \mathrm{e}-02$ & $1.667 \mathrm{e}-02$ & 0.91 & 52537 & 1454 & 49 & $2.507 \mathrm{e}-02$ & $1.883 \mathrm{e}-02$ & 0.751 \\
196074 & 2310 & 121 & $1.286 \mathrm{e}-02$ & $4.921 \mathrm{e}-03$ & 0.38 & 121978 & 2357 & 73 & $2.039 \mathrm{e}-02$ & $3.193 \mathrm{e}-02$ & 1.566 \\
409573 & 3744 & 181 & $8.943 \mathrm{e}-03$ & $7.127 \mathrm{e}-03$ & 0.80 & 264748 & 3718 & 109 & $1.378 \mathrm{e}-02$ & $5.095 \mathrm{e}-03$ & 0.370 \\
942465 & 6196 & 271 & $6.017 \mathrm{e}-03$ & $4.767 \mathrm{e}-03$ & 0.79 & 631452 & 5786 & 163 & $9.734 \mathrm{e}-03$ & $8.891 \mathrm{e}-03$ & 0.913 \\
2135099 & 10326 & 406 & $4.753 \mathrm{e}-03$ & $3.706 \mathrm{e}-03$ & 0.78 & 1506529 & 9258 & 244 & $7.409 \mathrm{e}-03$ & $4.234 \mathrm{e}-03$ & 0.572 \\
4678474 & 15715 & 609 & $3.101 \mathrm{e}-03$ & $3.128 \mathrm{e}-03$ & 1.01 & 3610055 & 15167 & 366 & $4.772 \mathrm{e}-03$ & $3.673 \mathrm{e}-03$ & 0.770 \\
10179407 & 24309 & 913 & $2.231 \mathrm{e}-03$ & $2.240 \mathrm{e}-03$ & 1.00 & 8109271 & 25747 & 549 & $3.314 \mathrm{e}-03$ & $3.374 \mathrm{e}-03$ & 1.018 \\
\hline
\end{tabular}

Table 4.1: Effectivity indices for the goal quantity 4.4 for different values of $\varepsilon$.

the robustness of our adaptive algorithm with respect to the small perturbation parameter $\varepsilon$.

To finish this section, we illustrate in Fig. 4.2 some stabilized solution profiles as well as adaptive spatial meshes after the last DWR loop at selected time points. Comparing the stabilized solution profile obtained on an adaptive refined mesh (cf. Fig. 4.2d) with its unstabilized counterpart obtained on an uniform refined mesh (cf. Fig. 4.1 c), both computed at the final time point $T$, we point out that the occuring spurious oscillations are strongly reduced. Furthermore, no smearing effects are observed, comparing the solution profiles of the hill at the respective time points. Considering the underlying spatial meshes, we note that the total number of the spatial cells is more or less equal during the whole time period, but the arrangement differs depending on the related position of the hill. Thus, for the chosen target functional (4.4) the spatial mesh runs as expected with the rotation of the hill in a synchronous way. In addition, we note that the mesh refinement is slightly weaker at the final time point. This is due to the error propagation of the underlying problem which is captured by the dual weights in the error estimate. This effect is in good agreement to the results obtained for the heat equation in [3, p. 122].

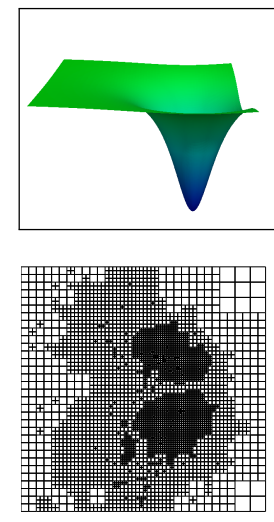

(a)
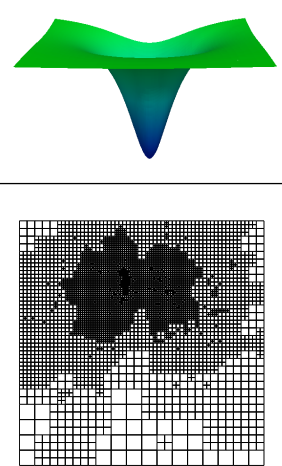

(b)

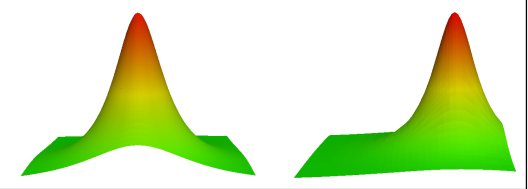

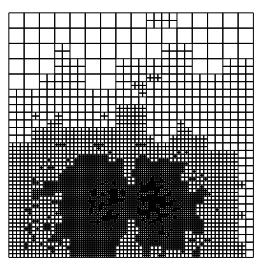

(c)

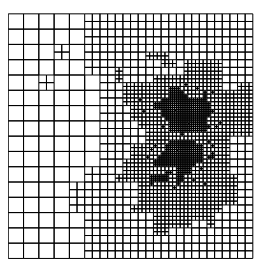

(d)

Figure 4.2: Stabilized solution profiles and related adaptive spatial meshes after 9 DWR loops at time points $t_{n}=0(\mathrm{a}), t_{n}=0.25(\mathrm{~b}), t_{n}=0.75(\mathrm{c})$ and $t_{n}=1$ (d) for $\varepsilon=10^{-6}$. 


\section{Conclusions and Outlook}

In this work we presented an adaptive solution algorithm for SUPG stabilized finite element approximations of time-dependent convection-diffusion-reaction equations. The underlying approach is based on the Dual Weighted Residual method for goal-oriented error control. A first dualize and then stabilize philosophy was applied for combining the space-time adaptation process in the course of the DWR approach with the stabilization of the finite element techniques. We used a higher-order finite element approximation in space and time in order to compute the dual solution. In numerical experiments we could prove that spurious oscillations that typically arise in numerical approximations of convection-dominated problems could be reduced significantly. Robust effectivity indices close to one were obtained for different values of the diffusion coefficient. It was shown that the DWR based adaptivity is no ballast on the way to solve convection-dominated problems. Conversely, it offers potential for further improvements in handling those problems.

We note that recent results in post-processing variational time discretization schemes (cf., e.g., [5, 1]) allow the computation of improved solutions admitting an additional order of convergence for the discretization in time by negligible computational costs, and thus offer further potential for reducing the costs of computing the dual solution.

\section{Acknowledgement}

We acknowledge Uwe Köcher for his help in the design and implementation of the underlying software DTM++/dwr-diffusion; cf. [16].

\section{References}

[1] Ahmed, N., John, V.: Adaptive time step control for higher order variational time discretizations applied to convection-diffusion equations. Comput. Methods Appl. Mech. Engrg. 285, 83-101 (2015)

[2] Alzetta, G., Arndt, D., Bangerth, W., Boddu, V., Brands, B., Davydov, D., Gassmoeller, R., Heister, T., Heltai, L., Kormann, K., Kronbichler, M., Maier, M., Pelteret, J.-P., Turcksin, B., Wells, D.: The deal.II Library, Version 9.0. J. Numer. Math., accepted (2018)

[3] Bangerth, W., Rannacher, R.: Adaptive finite element methods for differential equations. Birkhäuser, Basel (2003)

[4] Bause, M., Schwegler, K.: Analysis of stabilized higher order finite element approximation of nonstationary and nonlinear convection-diffusionreaction equations. Comput. Methods Appl. Mech. Engrg. 209-212, 184$196(2012)$

[5] Bause, M., Köcher, U., Radu, F. A., Schieweck, F.: Post-processed Galerkin approximation of improved order for wave equations. Math. Comput., submitted (2018), arXiv:1803.03005 
[6] Becker, R.: An optimal-control approach to a posteriori error estimation for finite element discretizations of the Navier-Stokes equations, East-West J. Numer. Math. 8, 257-274 (2000)

[7] Becker, R., Rannacher, R.: Weighted a posteriori error control in FE methods. In: Bock, H. G. et al. (eds.) ENUMATH 97. Proceedings of the 2nd European Conference on Numerical Mathematics and Advanced Applications, pp. 621-637. World Scientific, Singapore (1998)

[8] Becker, R., Rannacher, R.: An optimal control approach to a posteriori error estimation in finite element methods. In: Iserles, A. (ed.) Acta Numer., vol. 10, pp. 1-102. Cambridge University Press (2001)

[9] Besier, M., Rannacher, R.: Goal-oriented space-time adaptivity in the finite element Galerkin method for the computation of nonstationary incompressible flow. Int. J. Num. Methods Fluids 70(9), 1139-1166 (2012)

[10] Brooks, A. N., Hughes, T. J. R.: Streamline upwind/Petrov-Galerkin formulations for convection dominated flows with particular emphasis on the incompressible Navier-Stokes equations. Comput. Methods Appl. Mech. Engrg. 32(1-3), 199-259 (1982)

[11] Bruchhäuser, M. P., Schwegler, K., Bause, M.: Numerical study of goaloriented error control for stabilized finite element methods. In Apel, T. et al. (eds.) Advanced Finite Element Methods with Applications, Lecture Notes in Computational Science and Engineering, accepted (2018), pp. 1-19. arXiv:1803.10643

[12] Hughes, T. J. R., Brooks, A. N.: A multidimensional upwind scheme with no crosswind diffusion. In: Hughes, T. J. R. (eds.) Finite Element Methods for Convection Dominated Flows, AMD, vol. 34, pp. 19-35. Amer. Soc. Mech. Engrs. (ASME) (1979)

[13] John, V., Novo, J.: Error analysis of the SUPG finite element discretization of evolutionary convection-diffusion-reaction equations. SIAM J. Numer. Anal. 49(3), 1149-1176 (2011)

[14] John, V., Knobloch, P., Novo, J.: Finite elements for scalar convectiondominated equations and incompressible flow problems: a never ending story? Comput. Vis. Sci., https://doi.org/10.1007/s00791-018-0290-5, 1$17(2018)$

[15] John, V., Schmeyer, E.: Finite element methods for time-dependent convection-diffusion-reaction equations with small diffusion. Comput. Methods Appl. Mech. Engrg. 198, 173-181 (2009)

[16] Köcher, U, Bruchhäuser, M. P., Bause, M.: Efficient and scalable data structures and algorithms for goal-oriented adaptivity of space-time FEM codes. In progress, 1-6 (2018).

[17] Roos, H.-G., Stynes, M., Tobiska, L.: Robust Numerical Methods for Singularly Perturbed Differential Equations. Springer, Berlin (2008) 